\title{
Analysis of lignin content in alkaline treated hemp fibres: thermogravimetric studies and determination of kinetics of different decomposition steps
}

\author{
Gabriela Mijas ${ }^{\mathrm{a}}$, Albert Manich ${ }^{\mathrm{b}}$, Manuel-José Lis ${ }^{\mathrm{a}, \mathrm{c}}$, Marta Riba- \\ Moliner $^{\mathrm{a}, \mathrm{d}}$, Inés Algaba ${ }^{\mathrm{e}}$, Diana Cayuela ${ }^{\mathrm{a}, \mathrm{d}^{*}}$ \\ ${ }^{a}$ Terrassa Institute of Textile Research and Industrial Cooperation (INTEXTER), \\ Universitat Politècnica de Catalunya (UPC), Terrassa, Spain \\ ${ }^{b}$ Institute for Advanced Chemistry of Catalonia (IQAC), Spanish National Research \\ Council (CSIC), Barcelona, Spain
}

'Department of Chemical Engineering (EQ), Terrassa School of Industrial, Aerospace and Audiovisual Engineering (ESEIAAT), Universitat Politècnica de Catalunya (UPC), Terrassa, Spain

${ }^{d}$ Department of Materials Science and Engineering (CEM), Terrassa School of Industrial, Aerospace and Audiovisual Engineering (ESEIAAT), Universitat Politècnica de Catalunya (UPC), Terrassa, Spain

${ }^{e}$ Department of Statistics and Operations Research (EOI), Terrassa School of Industrial, Aerospace and Audiovisual Engineering (ESEIAAT), Universitat Politècnica de Catalunya (UPC), Terrassa, Spain

*corresponding author: diana.cayuela@upc.edu 


\title{
Analysis of lignin content in alkaline treated hemp fibres: thermogravimetric studies and determination of kinetics of different decomposition steps
}

\begin{abstract}
Hemp (Cannabis sativa L.) is a plant of which, its ease of cultivation, structure and properties, provide it great potential for industrial applications; nevertheless, the unfavourable content of lignin complicates its processing, especially in the textile area. Thus, an appropriate knowledge of the delignification process would lead to treating hemp fibres as cotton fibres. In this work, hemp has been treated with an alkaline liquor $[\mathrm{NaOH} 1 \mathrm{M}]$ at $110{ }^{\circ} \mathrm{C}$ during different times and afterwards, the substrates were chemically characterised in order to determine their lignin content and also analysed by thermogravimetry (TGA). Through the correlation analysis, the relationship between lignin content, onset temperature of cellulose decomposition and loss of mass at the different decomposition steps were established. A hyperbolic kinetic model to explain the influence of alkaline treatment time on lignin content and also on the loss of mass at the four steps of decomposition of the samples by TGA up to $600^{\circ} \mathrm{C}$ has been developed. Although it has been found that the degradation of lignin occurred across the entire temperature range, results have shown that the influence of treatment time has become clearly relevant on lignin content, $\operatorname{Step}_{2}\left(180.5{ }^{\circ} \mathrm{C}-273.5{ }^{\circ} \mathrm{C}\right)$ where a fraction of 10 to $16 \%$ of lignin was degraded, and $\mathrm{Step}_{3} 273.5^{\circ} \mathrm{C}-396.5^{\circ} \mathrm{C}$ ) where cellulose was mainly decomposed and $20 \%$ of lignin was also degraded.
\end{abstract}

Keywords: Hemp, lignin, alkaline treatment, thermogravimetric analysis (TGA), kinetics

\section{Introduction}

In recent decades, advances in studies focused on the biodegradation and sustainability of renewable resources have promoted a change in the social paradigm. This has attracted considerable attention to vegetal fibres as a raw material for the future, as well as a source of other components for new agrochemical and energy products. ${ }^{[1]}$ The production of vegetal fibres will help to improve the agricultural balance of the planet and reduce the deficit of cellulose pulp growing with the world population. ${ }^{[2]}$ 
Hemp (Cannabis sativa L.) is a plant of the Cannabaceae family, which has been grown and used in several countries, habitats and altitudes. ${ }^{[3,4]}$ Its ability to root deeply entails low nitrogen demand without continuous irrigation and its uses in bioremediation, lead to significant environmental benefits compared to other competing arable crops, such as cotton. ${ }^{[5-7]}$

Hemp has a fibrous structure with evident applications due to its properties. It is found in the group of woody species, such as ramie and flax. It has a cellulose content (70-74\%), which is lower than that of cotton, in addition to hemicellulose (18-23\%), pectins $(0.9 \%)$, lignin (4-6\%), water-soluble substances and a certain amount of natural pigments $(0.8 \%) .{ }^{[8,9]}$ Lignin creates mechanical incrustations in sections of amorphous cellulose and its presence in both, the elementary fibre and in the layers of the technical fibre, makes them stiffer, more breakable, and reduces their elementarization and spinnability. ${ }^{[10-12]}$ A proper understanding of the delignification would help to cottonize hemp, that is, to separate the fibre bundles into elemental fibres by removing the lignin that holds them together. After this process, hemp fibres can be spun with equipment intended for cotton. ${ }^{[4,10,13,14]}$

The process of cottonizing hemp fibres has been addressed from mechanical, chemical and enzymatic viewpoints that in combination with pre-treatments such as steaming and heating, ease the elimination of lignin, resulting in a modification of the structure of the fibres, specific surface, crystallinity, thermal stability, mechanical properties, moisture absorption and desorption. ${ }^{[10,15-22]}$ The control of these properties is fundamental to obtain suitable hemp fibres, alone or blended, for textile process, in order to produce substrates for clothing and household applications.

Kinetics and mechanism of delignification of hemp can be described by alkaline pulping, which consists essentially in the treatment of wood with an alkaline liquor and 
heat in a pressure resisting vessel. As it can be seen in Figure $1^{[23,24]}$, the wood fibre constituents were assumed to be present in the fibre-bound liquid, and the cooking liquor by chemical mass transfer phenomena between the reaction components such as, water, hydroxide ion, sodium ion, etc., extracts most of the lignin, pentosan, and other non- cellulosic materials, leaving the desired cellulose. Lignin dissolution can be described as a transfer process of molecules from the insoluble fibre wall to the fibrebound liquid. ${ }^{[24-26]}$ Both the soda and kraft pulping of non-wood and wood chips have been studied and some delignification models with different complexities have been described in literature. In the pulping process, initial, bulk and residual delignification stages can be distinguished. In the initial stage, hemicelluloses are deacetylated and a considerable amount is dissolved, while a small amount of lignin is removed. In the bulk stage, most of lignin is degraded and dissolved in the cooking liquor and, a smaller amount of the remaining hemicelluloses is dissolved or degraded, while the degree of polymerization of the cellulose decreases. During the residual stage, removal of lignin is very slow, while cellulose and remaining hemicellulose are decomposed further. ${ }^{[27,28]}$

\section{Figure 1}

De Groot et al. ${ }^{[27]}$ carried out an alkaline delignification of hemp woody core to develop an efficient and non-polluting process. Shavings of hemp woody core were delignified isothermally at different temperatures with $1 \mathrm{M}$ sodium hydroxide in a flowthrough reactor. They modelled the alkaline delignification kinetics restricted to the bulk and the rest of stages, described with two simultaneous first order reactions. Correia, Roy and Goel ${ }^{[29]}$ studied the delignification kinetics of Canadian hemp fibre through series of laboratory-scale micro-pulping experiments. The pulping liquors had an active alkali of $15 \%$ and $18 \%$ as $\mathrm{Na}_{2} \mathrm{O}$ from $\mathrm{NaOH}$ at various cooking temperatures in stainless-steel reactors. The calculated activation energies for hemp, based on first 
order kinetics, showed that the reaction is more energy efficient compared to other wood species. Dang and Nguyen ${ }^{[30]}$ described the heterogenous nature of alkaline pulping kinetics by a model based on the power law of growth and Avrami's concepts in nuclei growth taking into account the effects of alkali concentration and temperature. They tested the developed model against published data obtained from pulping of thin hemp woody core in isothermal flow-through reactors. The final form of the model could be represented by a first order rate equation with a time-dependent rate constant. Heterogeneous kinetic model was found to accurately predicted delignification and xylan loss from hemp woody core.

Regarding the studies conducted by thermogravimetric analysis (TGA) of hemp fibres, Kabir et al. ${ }^{[31]}$ treated hemp fibres with alkali $(0,4,6,8,10 \% \mathrm{NaOH})$, acetyl (acetic acid and acetic anhydride) and silane (3\% siloxane) chemicals. Results showed that hemicellulose was degraded faster than cellulose and lignin. Cellulose exhibited better thermal stability and lignin was degraded in wide temperature ranges. Treated fibres revealed higher thermal stability compared to untreated fibre. This last conclusion was also found by Stevulova et al. ${ }^{[15]}$ and by Zhang and Zhang. ${ }^{[19]}$

The aim of this work is to study the relationship between lignin content, onset temperature of cellulose decomposition and loss of mass of the different decomposition steps given by the Thermogravimetric Analysis (TGA) through the application of correlation analysis. The kinetics of alkaline hemp delignification by using the residual lignin after chemical treatments, and the kinetics of the different decomposition steps by monitoring the loss of mass given by thermogravimetric analysis as a function of the time of treatment are also determined.

\section{Materials and Methods}

Plant fibrous raw material 
The raw material used was hemp (Cannabis sativa L.) fibre purchased from CELESA S.A. (Spain). Previously, fibres were carded and washed with a non-ionic surfactant (Hostapal UH Liq, Archroma), $1.7 \mathrm{~g} / \mathrm{L}$, liquor ratio $1: 30$ at $90 \pm 2{ }^{\circ} \mathrm{C}$ for 10 minutes and rinsed with deionized water at room temperature for 5 minutes, 3 times. Finally, hemp fibres were dried on filter papers. In order to improve fibre handling and to ensure homogeneity of the samples, $2 \mathrm{~kg}$ of washed hemp fibres were ground in a SR 100 (Retsch) to a size of $2 \mathrm{~mm}$ and subsequently, they were conditioned at $20^{\circ} \mathrm{C} \pm 2{ }^{\circ} \mathrm{C}$ and $65 \pm 4 \%$ R.H. for 24 hours.

\section{Lignins}

Lignosulfonates of low molecular, medium and high molecular weight, described in Table 1, were provided by Borregaard - Lignotech Ibérica S.A. (Spain) and used as comparison materials.

\section{Alkaline chemical treatment}

Chemical treatments of hemp were carried out in a Carousel 6 Plus reaction station (Radlyes) at $110^{\circ} \mathrm{C}, 400 \mathrm{rpm}$. In each reactor, $7.0 \pm 0.5 \mathrm{~g}$ of conditioned fibre and 105 $\mathrm{mL}$ of pulping liquor were placed. Fibre to liquor ratio was $1: 15$ and the liquor was made of $\mathrm{NaOH}$ (Scharlau) [40 g/L] and an anionic- non-ionic surfactant (Hostapal DTC, Archroma) $[1.5 \mathrm{~g} / \mathrm{L}]$. The chemical treatment of the hemp was performed during 5, 15, $30,45,60,90,120,180,240,360$ and 480 minutes. After the reaction time has elapsed, the samples were neutralized with acetic acid (Scharlau) $[0.1 \mathrm{M}]$, rinsed with deionized water three times and dried at $50{ }^{\circ} \mathrm{C} \pm 0.5^{\circ} \mathrm{C}$ for 24 hours.

\section{Lignin determination}

Lignin content (\%) in hemp fibres after the chemical treatment at different times was 
determined according to Tappi Standard T 222 om-2015 "Acid-insoluble lignin in wood and pulp". ${ }^{[32]}$ The percentage of lignin removed at each time was calculated based on the lignin content in untreated hemp.

Thermogravimetric analysis (TGA)

TGA were performed in a Mettler-Toledo TGA/SDTA 840 by using samples of about $10 \mathrm{mg}$ placed in completely open aluminium pans. Tests were conducted from $25^{\circ} \mathrm{C}$ to $600{ }^{\circ} \mathrm{C}$ at $10{ }^{\circ} \mathrm{C} / \mathrm{min}$ under a $\mathrm{N}_{2}$ flux of $60 \mathrm{~mL} / \mathrm{min}$. Experimental results were obtained with STARe Software 10.0 (Mettler Toledo), which allows to analyse TGA plots by determining derivatives, decomposition steps, thermal transition temperatures and loss of mass at the different steps.

\section{Statistical Modelling}

Experimental results were statistically analysed with Statgraphics Plus Software V.1 (Statistical Graphics Corporation and Manugistics, Inc.), to obtain the correlation analysis between the results in order to determine the existing relationships and, also to find the kinetic model to predict the evolution of lignin, loss of mass of the different decomposition steps and residue as a function of the treatment time by applying a nonlinear regression.

\section{Results and Discussion}

TGA plots of a sample of low molecular weight lignin and that of an untreated hemp are shown in Figure 2.

\section{Figure 2}

The four decomposition steps defined by the DTGA plots during the TGA of lignin and hemp were as follows. ${ }^{[31]}$ The three $\mathrm{T}_{\text {end }}$ temperatures indicated are the average of those 
obtained from hemp samples.

- First step from room temperature to $T_{\text {end1 }}\left(180.5^{\circ} \mathrm{C}\right)$ : Moisture and volatiles like waxes and lignin of very low molecular weight are mainly removed. Step $\mathrm{p}_{1}$ is the corresponding loss of mass.

- Second step from $\mathbf{T}_{\text {end1 }}$ to $\mathbf{T}_{\text {end2 }}\left(273.5^{\circ} \mathbf{C}\right)$ : Mainly pectin and lignin of lowmedium molecular weight are removed. Step 2 is the corresponding loss of mass.

- Third step from $\mathbf{T}_{\text {end2 }}$ to $\mathbf{T}_{\text {end3 }}\left(396.5^{\circ} \mathbf{C}\right)$ : Degradation of cellulose takes place besides that of lignin of medium-high molecular weight. Step ${ }_{3}$ is the corresponding loss of mass.

- Fourth step from $\mathbf{T}_{\text {end3 }}$ to $600{ }^{\circ} \mathbf{C}$ : Char derivatives from cellulose and lignin residues are formed. Step 4 is the corresponding loss of mass.

- Residue at $600{ }^{\circ} \mathrm{C}$ is tar and char formed products remaining at the end of the TGA.

The percentage loss of mass of low, medium and high molecular weight lignosulfonates obtained by TGA is shown in Table 2 .

Table 2

The percentage loss of mass of untreated hemp sample and of those treated chemically in the reactor according to the treatment time is shown in Table 3.

\section{Table 3}

\section{Correlation analysis}

The possible relationship between the lignin content (or lignin removed) and the results given by TGA has been analysed by a correlation analysis. The results corresponding to the untreated sample are highly detached from those of the 11 treated samples, which 
leads to high correlation coefficient although there is a weak relationship between variables. Consequently, only the relationships TGA results to lignin content (or lignin removed) with correlation coefficients greater than 0.9 have been considered. The results are shown in Table 4.

\section{Table 4}

Correlation analysis shows that the greater the lignin content, the higher the loss of mass of both $\mathrm{Step}_{2}$ and $\mathrm{Step}_{4}$, the lower that of $\mathrm{Step}_{3}$ and the presence of lignin causes the onset temperature of cellulose decomposition $\left(\mathrm{T}_{\text {on }}\right)$ to decrease. The results of Table 2 enable to consider that during $\mathrm{Step}_{2}$, low molecular weight lignins are removed, while during $\mathrm{Step}_{4}\left(400{ }^{\circ} \mathrm{C}-600{ }^{\circ} \mathrm{C}\right)$ more stable lignins are removed regardless their molecular weight. Step ${ }_{3}$ is mainly related to the cellulose decomposition: The greater the cellulose content, the lower the lignin content and, consequently, less amount of lignin will be removed from the sample at this step. The relationship between $T_{\text {on }}$ and lignin content suggests that the presence of lignin decreases the temperature at which the decomposition of cellulose begins. Otherwise, the greater the cellulose content, the higher both, the size of $\mathrm{Step}_{3}$ and the onset temperature of decomposition. Lignin impairs the thermal stability of cellulose.

As regards the relationship between the TGA results and the onset temperature of cellulose decomposition $\left(\mathrm{T}_{\mathrm{on}}\right)$, increases with the amount of cellulose (higher values of $\mathrm{Step}_{3}$ ) due to the reduction of lignin content, which is mainly decomposed in both $\mathrm{Step}_{2}$ and $\mathrm{Step}_{4}$. The relationships between the three steps show that the higher the $\mathrm{Step}_{2}$, the greater $\mathrm{Step}_{4}$ and the lower $\mathrm{Step}_{3}$, which concurs with the positive relationships between lignin content with both $\mathrm{Step}_{2}$ and $\mathrm{Step}_{4}$, and cellulose content with $\mathrm{Step}_{3}$. 
Kinetic modelling of lignin content and TGA results of loss of mass at the different steps and residue as a function of the reactor treatment time

The kinetics of lignin content (LC) and the variation of mass at each step as a function of the alkaline treatment time ( $\mathrm{Step}_{1}, \mathrm{Step}_{2}, \mathrm{Step}_{3}, \mathrm{Step}_{4}$ and Residue) can be modelled by using a hyperbolic model, ${ }^{[33]}$ which at time zero provides the initial mass of the untreated sample $m_{0}$, and over time, evolves towards a horizontal asymptote indicating the maximum mass variation due to the treatment reached at infinite time. If $m$ is the percentage loss or gain of mass at each stage depending on the treatment time in minutes, the model is as follows.

$$
m(\%)=A+\frac{B}{\sqrt{\text { time }+C}}
$$

The initial value of the untreated sample mass $m_{0}$ is obtained from equation (1) for $t=$ 0 .

$$
m_{0}(\%)=A+\frac{B}{\sqrt{C}}
$$

The final value of mass at infinite time (asymptote) of treatment corresponds to the constant A.

$$
m_{\infty}(\%)=A
$$

The $50 \%$ of the maximum variation of mass due to the treatment is obtained as follows.

$$
\Delta m_{1 / 2}(\%)=\frac{m_{0}-m_{\infty}}{2}
$$

Which means that the mass content will be $m_{1 / 2}=m_{0}+\Delta m_{1 / 2}$ and when replaced in the model, allows to determine the time of half variation of mass $t_{1 / 2}$ according to the 
equation (5).

$$
t_{1 / 2}(\min )=\left(\frac{B}{m_{1 / 2}-A}\right)^{2}-C
$$

By calculating the derivative of the model, the initial rate of mass variation at $\mathrm{t}=0$ can be calculated.

$$
m_{0}^{\prime}=-0.5 * B * C^{-1.5}
$$

The theoretical kinetic model and their parameters are shown in Figure 3.

\section{Figure 3}

The parameters of the models fitted to the lignin content and to the different steps and residue given by the TGA have been obtained by means of nonlinear regression. Table 5 shows the parameters $\mathrm{A}, \mathrm{B}$ and $\mathrm{C}$ of the model, coefficient $\mathrm{R}^{2}$, characteristic values of mass $m_{0}, m_{1 / 2}, m_{\infty}$, initial rate of mass variation $m_{0}^{\prime}$ and time of half variation of mass $\mathrm{t}_{1 / 2}$.

\section{Table 5}

The goodness of fit is evaluated by the signification of the coefficients B and C of the model and by coefficient of determination $\mathrm{R}^{2}$. Results in bold type indicate that coefficients are significant at $5 \%$, which means that the model shows satisfactorily the change of mass as a function of time. Additionally, the three models containing both B and $\mathrm{C}$ coefficients, have $\mathrm{R}^{2}$ values greater than $97 \%$, which means models account for the $97 \%$ of quadratic mass variation over time and the experimental error is lower than $3 \%$.

Plots of the models fitted to the lignin content in samples and to the different steps and residue of TGA results are shown in Figure 4. 


\title{
Figure 4
}

Relationship between lignin content (LC) and TGA results of Step and Step $_{3}$

Based on the results of the best fits, it seems that it may be convenient to study the relationship between the results given by TGA at $\mathrm{Step}_{2}$ and $\mathrm{Step}_{3}$ with the lignin content in the sample. A linear regression shown in figure 5 between both $\mathrm{Step}_{2}$ and $\mathrm{Step}_{3}$ and LC, predicts the lignin content in the sample based on the loss of mass at both steps.

\section{Figure 5}

\begin{abstract}
Alkaline delignification could influence the decomposition of other components of hemp in addition to lignin. However, it seems that these remains may be lost in stages prior to stage 3 probably due to their molecular weight after alkaline hydrolysis, which could explain the reason why they do not conform to linearity.
\end{abstract}

On the other hand, Table 4 shows that the higher the lignin content, the smaller the Step 3, and consequently the loss of mass due to cellulose decomposition. Additionally, it must be considered that the lignin content in the fibre is around $6 \%$, therefore, $6 \%$ of mass lost in the lignin decomposition in Step 3 which is around $20 \%$ (Table 2), would represent $1.2 \%$ of the loss observed in the Step 3, in which the losses of mass reach $70 \%$. So, it seems that the presence of lignin practically does not distort the losses in the hemp measured in the Step 3.

Table 4 and Table 3 also show that the presence of lignin (therefore, it must be its decomposition) could favour that cellulose decomposition $\left(\mathrm{T}_{\mathrm{on}}\right)$ starts sooner $(\mathrm{r}=$ 0.916) so, the more lignin is in the fibre, the sooner the cellulose decomposition begins, which is clearly observed when comparing delignified samples with untreated hemp. 


\section{Influence of treatment time on Residue}

As regards the model fitted to Residue, neither B nor C coefficients are significant and the coefficient of determination $\mathrm{R}^{2}$ is lower than $77 \%$, which means that the experimental error of the quadratic variation is greater than $33 \%$. Consequently, it can be expected that time has not any significant effect on Residue, which will be reflected in a normal distribution of the results.

The application of Grubbs criterion for the determination of anomalous values in a normal distribution yields the following results: 12 values between 16.49 and $20.37 \%$ with a sample mean of $17.46 \%$. The standardized distance of each result compared to the sample mean shows that the highest value (which corresponds to the residue of the untreated sample) gives a result of 2.77 , exceeding the allowable limit for this sample size (2.41). Thus, the probability that the Residue of the untreated sample belongs to the normal distribution is $0.26 \%$, so it can be assumed that it does not belong to this distribution.

In contrast, the application of Grubbs criterion to the treated samples gives the following results: 11 values between 16.49 and $18.15 \%$ with a sample mean of 17.19 $\%$. The standardized distance of the highest difference yields a parameter of 1.76 below the limit for this sample size (2.36), so it can be stated that these values follow a normal distribution with a probability of $65.04 \%$. The confidence intervals for the sample mean of 17.19 at $95 \%$ are between 16.83 and $17.56 \%$. The A values of the Steps 1 to 4 accounts for a loss of mass of $82.57 \%$ resulting in a Residue of $17,43 \%$ which are included in the confidence interval of the treated sample mean.

\section{Conclusions}

The influence of lignin when comparing the degradation of the untreated hemp with that of the delignified hemp has been evaluated. When analysing the degradation stages of 
low, medium and high molecular weight lignins, it has been seen that the degradation of lignin occurs in all temperature zones and not only between $350{ }^{\circ} \mathrm{C}$ and $450{ }^{\circ} \mathrm{C}$ as mentioned in previous works. ${ }^{[34]}$

A hyperbolic kinetic model to explain the influence of alkaline treatment time on lignin content of hemp samples and also on the loss of mass of the four steps of decomposition of the samples by TGA up to $600{ }^{\circ} \mathrm{C}$ has been developed.

By using this hyperbolic model, the influence of time has become clearly relevant on the following results: a) Lignin content (LC), b) Step 2 , where a fraction of 10 to $16 \%$ of lignin was degraded, and c) Step 3 where cellulose was mainly decomposed. In this Step, more than $20 \%$ of lignin was also degraded.

The best linear regressions between lignin content and TGA results have been calculated and they were those given by Step 2 and $\mathrm{Step}_{3}$. The Residue was not influenced by the time of alkaline treatment. The Residue of the untreated sample was $20.37 \%$ while the treatment caused the Residue to decrease down to $17.19 \%$ regardless of the treatment time. Char components and tar derived from both lignin and cellulose which were present in the residue were not affected by the time of treatment.

\section{Acknowledgements}

Authors gratefully acknowledge the financial support for this research from the Ministry of Science and Innovation of Spain (Project MAT2016-79352-R) and from the National Secretary of Higher Education, Science, Technology and Innovation of Ecuador (SENESCYT) for Doctoral's Fellowship (No. CZ02-000926-2018). Also, authors would like to thank to Masias Maquinaria for the carding of hemp fibres and Borregaard - Lignotech Ibérica SA for providing the lignosulfonates. Finally, authors acknowledge Mrs. Mercedes Simò for their support in the experimental work.

\section{References}

[1] Pereira, P. H. F.; Rosa, M. de F.; Cioffi, M. O. H.; Benini, K. C. C. de C.; Milanese, A. C.; Voorwald, H. J. C.; Mulinari, D. R. Vegetal Fibers in Polymeric 
Composites: A Review. Polímeros, 2015, 25 (1), 9-22.

https://doi.org/10.1590/0104-1428.1722.

[2] Kozlowski, R.; Baraniecki, P.; Barriga-Bedoya, J. Bast Fibres (Flax, Hemp, Jute, Ramie, Kenaf, Abaca). In Biodegradable and Sustainable Fibres; Blackburn, R. S., Ed.; Woodhead Publishing Series in Textiles; Woodhead Publishing: Cambridge, 2005; pp 36-88. https://doi.org/https://doi.org/10.1533/9781845690991.36.

[3] ElSohly, M. A.; Radwan, M. M.; Gul, W.; Chandra, S.; Galal, A. Phytochemistry of Cannabis Sativa L. In Phytocannabinoids: Unraveling the Complex Chemistry and Pharmacology of Cannabis sativa; Kinghorn, A. D., Falk, H., Gibbons, S., Kobayashi, J., Eds.; Springer International Publishing: Cham, 2017; pp 1-36. https://doi.org/10.1007/978-3-319-45541-9_1.

[4] Horne, M. R. L. Bast Fibres: Hemp Cultivation and Production. In Handbook of Natural Fibres - Volume 1:Types, properties and factors affecting breeding and cultivation; Kozłowski, R., Ed.; Woodhead Publishing Limited: Cambridge, 2012; p 620.

[5] Angelova, V.; Ivanova, R.; Delibaltova, V.; Ivanov, K. Bio-Accumulation and Distribution of Heavy Metals in Fibre Crops (Flax, Cotton and Hemp). Ind. Crops Prod., 2004, 19 (3), 197-205. https://doi.org/10.1016/j.indcrop.2003.10.001.

[6] Grabowska, L.; Baraniecki, P. Three Year Results on Utilization Soil Polluted by Copper Producing Industry for Cultivation of Industrial Crops. In Flax and Other Bast Plants Symp. Natural Fibres, Spec. Ed.; Poznan, 1997; pp 123-131.

[7] Karczewska, A.; Mocek, A.; Piotr, G.; Mleczek, M. Phytoremediation: Management of Environmental Contaminants, Volume 2. In Phytoremediation: Management of Environmental Contaminants; Ansari, A. A., Gill, S. S., Gill, R., Lanza, G. R., Newman, L., Eds.; Springer International Publishing: Cham, 2015; Vol. 2, pp 143-170. https://doi.org/10.1007/978-3-319-10969-5.

[8] Bismarck, A.; Baltazar-Y-Jimenez, A.; Sarikakis, K. Green Composites as Panacea? Socio-Economic Aspects of Green Materials. Environ. Dev. Sustain., 2006, 8 (3), 445-463. https://doi.org/10.1007/s10668-005-8506-5. 
[9] Shahzad, A. Hemp Fiber and Its Composites - a Review. J. Compos. Mater., 2012, 46 (8), 973-986. https://doi.org/10.1177/0021998311413623.

[10] Cierpucha, W.; Kozłowski, R.; Mańkowski, J.; Waśko, J.; Mańkowski, T. Applicability of Flax and Hemp as Raw Materials for Production of Cotton-like Fibres and Blended Yarns in Poland. Fibres Text. East. Eur., 2004, 12 (3), 13-18.

[11] D’Almeida, A. L. F. S.; Barreto, D. W.; Calado, V.; D’Almeida, J. R. M. Thermal Analysis of Less Common Lignocellulose Fibers. J. Therm. Anal. Calorim., 2008, 91 (2), 405-408. https://doi.org/10.1007/s10973-007-8606-6.

[12] Aishwarya Anand, A. S.; Ramakrishnan, G.; Srinivasan, J. A Critical Review on: Development of An Absorbent Fitness Wear Using Hemp Fibre. J. Fibre To Finish, 2018, 57, 27-34.

[13] Sedelnik, N. Properties of Hemp Fibre Cottonised by Biological Modification of Hemp Hackling Noils. Fibres Text. East. Eur., 2004, 12 (1), 58-60.

[14] Zhang Jinqiu; Zhang Jianchun. Effect of Refined Processing on the Physical and Chemical Properties of Hemp Bast Fibers. Text. Res. J., 2010, 80 (8), 744-753. https://doi.org/10.1177/0040517509342317.

[15] Stevulova, N.; Cigasova, J.; Estokova, A.; Terpakova, E.; Geffert, A.; Kacik, F.; Singovszka, E.; Holub, M. Properties Characterization of Chemically Modified Hemp Hurds. Materials (Basel)., 2014, 7 (12), 8131-8150. https://doi.org/10.3390/ma7128131.

[16] Mwaikambo, L. Y.; Ansell, M. P. Chemical Modification of Hemp, Sisal, Jute, and Kapok Fibers by Alkalization. J. Appl. Polym. Sci., 2002, 84, 2222-2234. https://doi.org/10.1002/app.10460.

[17] Bourmaud, A.; Morvan, C.; Baley, C. Importance of Fiber Preparation to Optimize the Surface and Mechanical Properties of Unitary Flax Fiber. Ind. Crops Prod., 2010, 32 (3), 662-667. https://doi.org/10.1016/j.indcrop.2010.08.002.

[18] Islam, M. S.; Pickering, K. L.; Foreman, N. J. Influence of Hygrothermal Ageing on the Physico-Mechanical Properties of Alkali Treated Industrial Hemp Fibre Reinforced Polylactic Acid Composites. J. Polym. Environ., 2010, 18 (4), 696704. https://doi.org/10.1007/s10924-010-0225-9. 
[19] Zhang, H.; Zhang, L. M. Improving the Dyeing Properties and Softness of Hemp Fabric Using Chitosan and Epoxy Modified Silicone Oil. J. Text. Inst., 2010, 101 (9), 849-857. https://doi.org/10.1080/00405000902935015.

[20] George, M.; Mussone, P. G.; Alemaskin, K.; Chae, M.; Wolodko, J.; Bressler, D. C. Enzymatically Treated Natural Fibres as Reinforcing Agents for Biocomposite Material: Mechanical, Thermal, and Moisture Absorption Characterization. $J$. Mater. Sci., 2016, 51 (5), 2677-2686. https://doi.org/10.1007/s10853-015-9582Z.

[21] Nilsson, D.; Svennerstedt, B.; Wretfors, C. Adsorption Equilibrium Moisture Contents of Flax Straw, Hemp Stalks and Reed Canary Grass. Biosyst. Eng., 2005, 91 (1), 35-43. https://doi.org/10.1016/j.biosystemseng.2005.02.010.

[22] Moya, R.; Tenorio, C.; Torres, J. D. C. Steaming and Heating Dipteryx Panamensis Logs from Fast-Grown Plantations: Reduction of Growth Strain and Effects on Quality. For. Prod. J., 2021, 71 (1), 3-10. https://doi.org/10.13073/FPJ-D-20-00041.

[23] Fearon, O.; Kuitunen, S.; Ruuttunen, K.; Alopaeus, V.; Vuorinen, T. Detailed Modeling of Kraft Pulping Chemistry. Delignification. Ind. Eng. Chem. Res., 2020, 59 (29), 12977-12985. https://doi.org/10.1021/acs.iecr.0c02110.

[24] Tarvo, V.; Lehtimaa, T.; Kuitunen, S.; Alopaeus, V.; Vuorinen, T.; Aittamaa, J. A Model for Chlorine Dioxide Delignification of Chemical Pulp. J. Wood Chem. Technol., 2010, 30 (3), 230-268. https://doi.org/10.1080/02773810903461476.

[25] Larocque, G. L.; Maass, O. The Mechanism of the Alkaline Delignification of Wood. Can. J. Res., 1941, $19 b$ (1), 1-16. https://doi.org/10.1139/cjr41b-001.

[26] de Groot, B.; van Dam, J. E. G.; van 't Riet, K. Alkaline Pulping of Hemp Woody Core: Kinetic Modelling of Lignin, Xylan and Cellulose Extraction and Degradation. Holzforschung, 1995, 49 (4), 332-342. https://doi.org/10.1515/hfsg.1995.49.4.332.

[27] de Groot, B.; van Dam, J. E. G.; van der Zwan, R. P.; van 't Riet, K. Simplified Kinetic Modelling of Alkaline Delignification of Hemp Woody Core. Holzforschung, 1994, 48 (3), 207-214. https://doi.org/10.1515/hfsg.1994.48.3.207. 
[28] Kondo, R.; Sarkanen, K. V. Kinetics of Lignin and Hemicellulose Dissolution during the Initial Stage of Alkaline Pulping. Holzforschung, 1984, 38 (1), 31-36. https://doi.org/10.1515/hfsg.1984.38.1.31.

[29] Correia, F.; Roy, D. N.; Goel, K. Chemistry and Delignification Kinetics of Canadian Industrial Hemp. J. Wood Chem. Technol., 2001, 21 (2), 97-111. https://doi.org/10.1081/WCT-100104221.

[30] Dang, V.; Nguyen, K. L. Characterisation of the Heterogeneous Alkaline Pulping Kinetics of Hemp Woody Core. Bioresour. Technol., 2006, 97 (12), 1353-1359. https://doi.org/10.1016/j.biortech.2005.07.008.

[31] Kabir, M. M.; Wang, H.; Lau, K. T.; Cardona, F. Effects of Chemical Treatments on Hemp Fibre Structure. Appl. Surf. Sci., 2013, 276, 13-23. https://doi.org/10.1016/j.apsusc.2013.02.086.

[32] Technical Association of the Pulp and Paper Industry. TAPPI T 222 Om-15. Acid-Insoluble Ligning in Wood and Pulp; TAPPI Press: Atlanta, 2011.

[33] Ratkowsky, D. A. Handbook of Nonlinear Regression Models.; M. Dekker: New York, 1990. https://doi.org/10.2307/2347928.

[34] Beall, F. C.; Eickner, H. W. Thermal Degradation of Wood Components: A Review of the Literature; Forest Products Laboratory: Madison, 1970. 
Figure 1. Alkaline pulping of hemp. The reactions between the cooking liquor (water, hydroxide ion, sodium ion, etc.) and reactive lignocellulosic constituents dissolve some of these components resulting in openings that allow the chemicals to penetrate the hemp structure

$$
106 \times 69 \mathrm{~mm}(300 \times 300 \mathrm{DPI})
$$


Figure 2. TGA and DTGA plots of samples of a) low molecular weight lignosulfonate and b) untreated hemp. The DTGA plots enable to define four decomposition steps ranging between room temperature and the successive $T_{\text {end }}$ temperatures up to $600^{\circ} \mathrm{C}$. The loss of mass at the different steps is evaluated. Two temperatures, onset temperature of cellulose decomposition $\mathrm{T}_{\text {on }}$ and that at which the cellulose decomposition attains its maximum $\mathrm{T}_{\text {peak } 3}$ are given 
Figure 3: Theoretical kinetic model of mass variation as a function of the treatment time in the reactor. Representation of the model parameters $A, B$ and $C$, and characteristic values of mass $m_{0}, m_{1 / 2}, m_{\infty}$, initial rate of mass variation $m^{\prime} 0$ and time of half variation of mass $t_{1 / 2}$ 


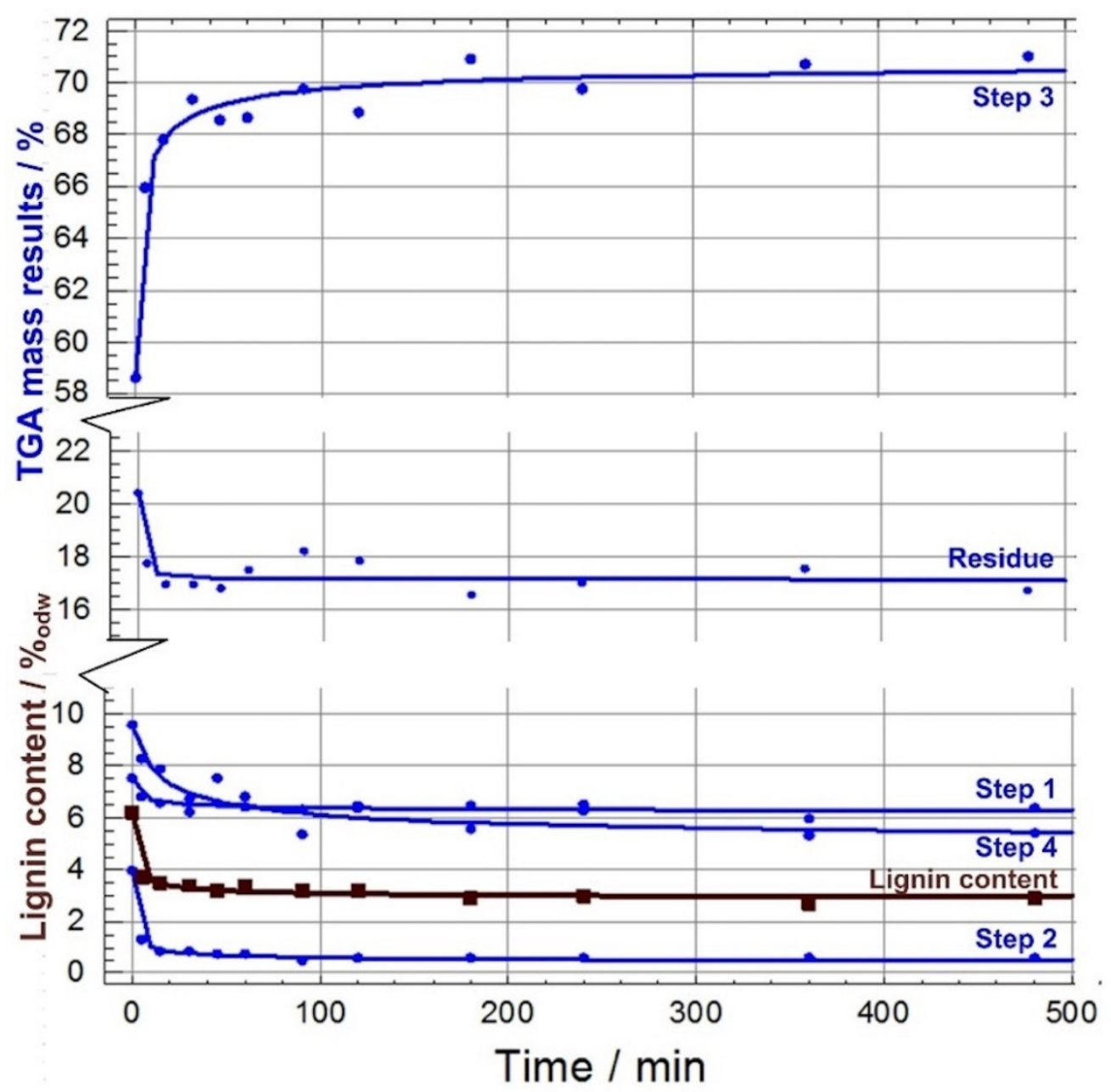

Figure 4: Kinetic models fitted to lignin content and to variation of mass at the different steps and residue given by TGA as a function of the alkaline treatment time

$99 \times 99 \mathrm{~mm}(300 \times 300$ DPI $)$ 
Figure 5: a) Linear regression between $\mathrm{Step}_{2}$ and lignin content and b) linear regression between Step3 and lignin content 
Table 1: Lignosulfonates used as comparison materials

\begin{tabular}{|c|c|c|c|}
\hline \multirow{2}{*}{ Component } & \multicolumn{3}{|c|}{ Lignosulfonates } \\
\hline & Low molecular weight & Medium molecular weight & High molecular weight \\
\hline $\begin{array}{l}\text { Dry matter (\% obtained by } \\
\left.\text { drying in oven at } 105^{\circ} \mathrm{C}\right)\end{array}$ & 55.7 & 52.1 & 52.2 \\
\hline $\begin{array}{c}\text { Ashes at } 850^{\circ} \mathrm{C}(\% \text { dry } \\
\text { matter basis) }\end{array}$ & 9.7 & 15.5 & 12.1 \\
\hline $\begin{array}{l}\text { Sugars (HPLC, \% dry } \\
\text { matter basis) }\end{array}$ & 22.9 & 4.3 & 6.4 \\
\hline
\end{tabular}


Table 2: TGA results of the percentage loss of mass of low, medium and high molecular weight lignosulfonates at the different steps of decomposition, Step ${ }_{1}$ (up to $180.5^{\circ} \mathrm{C}$ ), $\mathrm{Step}_{2}$ (up to $273.5{ }^{\circ} \mathrm{C}$ ), $\mathrm{Step}_{3}$ (up to $396.5{ }^{\circ} \mathrm{C}$ ), $\mathrm{Step}_{4}$ up to $600{ }^{\circ} \mathrm{C}$ and Residue at $600{ }^{\circ} \mathrm{C}$

\begin{tabular}{cccccc}
\hline Molecular weight & Step $_{\mathbf{1}}(\mathbf{\%})$ & Step $_{\mathbf{2}}(\mathbf{\%})$ & Step $_{\mathbf{3}}(\mathbf{\%})$ & Step $_{\mathbf{4}}(\mathbf{\%})$ & Residue (\%) \\
\hline Low & 16.09 & 16.38 & 21.47 & 10.24 & 35.82 \\
Medium & 8.86 & 13.25 & 22.68 & 9.28 & 45.93 \\
High & 8.56 & 10.47 & 23.06 & 9.78 & 48.12 \\
\hline
\end{tabular}


Table 3: TGA results of percentage loss of mass of hemp samples according to the treatment time of treatment in a reactor. The onset temperature of cellulose decomposition $\left(\mathrm{T}_{\mathrm{on}}\right)$, at which the maximum decomposition rate is attained $\left(\mathrm{T}_{\text {peak } 3}\right)$, and the percentages of lignin content on dry weight (o.d.w.) and lignin removed according to the time of treatment are also included

\begin{tabular}{|c|c|c|c|c|c|c|c|c|c|}
\hline $\begin{array}{l}\text { Time } \\
(\min )\end{array}$ & $\begin{array}{l}\text { Step }_{1} \\
(\%)\end{array}$ & $\begin{array}{l}\text { Step }_{2} \\
(\%)\end{array}$ & $\begin{array}{l}\text { Step }_{3} \\
(\%)\end{array}$ & $\begin{array}{l}\text { Step }_{4} \\
(\%)\end{array}$ & $\begin{array}{c}\text { Residue } \\
\text { (\%) }\end{array}$ & $\begin{array}{l}\mathrm{T}_{\text {on }} \\
\left({ }^{\circ} \mathrm{C}\right)\end{array}$ & $\begin{array}{l}\mathbf{T}_{\text {peak3 }} \\
\left({ }^{\circ} \mathrm{C}\right)\end{array}$ & $\begin{array}{c}\text { Lignin content } \\
\text { (\% odw) }\end{array}$ & $\begin{array}{c}\text { Lignin removed } \\
\text { (\%) }\end{array}$ \\
\hline 0 & 9.54 & 3.95 & 58.65 & 7.49 & 20.37 & 319.8 & 351.8 & 6.2 & 0 \\
\hline 5 & 8.25 & 1.30 & 65.95 & 6.81 & 17.68 & 333.8 & 353.7 & 3.7 & 40.5 \\
\hline 15 & 7.87 & 0.85 & 67.81 & 6.57 & 16.90 & 334.6 & 352.4 & 3.5 & 43.5 \\
\hline 30 & 6.20 & 0.81 & 69.39 & 6.69 & 16.90 & 332.9 & 352.7 & 3.4 & 45.4 \\
\hline 45 & 7.49 & 0.71 & 68.55 & 6.52 & 16.73 & 334.6 & 352.5 & 3.2 & 48.8 \\
\hline 60 & 6.79 & 0.71 & 68.67 & 6.40 & 17.43 & 333.7 & 353.7 & 3.4 & 46.1 \\
\hline 90 & 5.32 & 0.47 & 69.76 & 6.30 & 18.15 & 332.5 & 354.9 & 3.2 & 49.3 \\
\hline 120 & 6.34 & 0.55 & 68.86 & 6.44 & 17.81 & 333.3 & 351.3 & 3.2 & 49.3 \\
\hline 180 & 5.55 & 0.59 & 70.95 & 6.42 & 16.49 & 336.9 & 355.0 & 2.9 & 53.7 \\
\hline 240 & 6.25 & 0.58 & 69.76 & 6.47 & 16.93 & 334.9 & 352.9 & 3.0 & 51.1 \\
\hline 360 & 5.27 & 0.59 & 70.70 & 5.97 & 17.47 & 332.2 & 352.5 & 2.7 & 57.2 \\
\hline 480 & 5.39 & 0.56 & 71.04 & 6.37 & 16.64 & 333.9 & 353.6 & 2.9 & 53.6 \\
\hline
\end{tabular}


Table 4: TGA results with correlation coefficients with lignin content greater than 0.9

\begin{tabular}{|c|c|c|c|c|}
\hline $\mathrm{N}=12$ & Lignin content & $T_{\text {on }}$ & Step $_{4}$ & Step $_{3}$ \\
\hline$\overline{\text { Step }}_{2}$ & 0.980 & -0.929 & 0.894 & -0.963 \\
\hline$\overline{\text { Step }}_{3}$ & -0.979 & 0.874 & -0.914 & \\
\hline Step $_{4}$ & 0.924 & -0.736 & & \\
\hline $\mathbf{T}_{\text {on }}$ & -0.916 & & & \\
\hline
\end{tabular}


Table 5: Parameters A, B and C of the kinetic model fitted to the variation of lignin content (LC), and TGA results $\mathrm{Step}_{1}$ to $\mathrm{Step}_{4}$ and Residue with time, $\mathrm{R}^{2}$ coefficient, and kinetic characteristic values

\begin{tabular}{|c|c|c|c|c|c|c|c|c|}
\hline LC / TGA Results & $A=m_{\infty}(\%)$ & B $\left(\% \cdot \min ^{1 / 2}\right)$ & $C(\min )$ & $R^{2}(\%)$ & $m_{0}(\%)$ & $m_{1 / 2}(\%)$ & $t_{1 / 2}(\min )$ & $\mathrm{m}^{\prime}{ }_{0}(\% / \mathrm{min})$ \\
\hline Lignin content & 2.86 & 2.31 & 0.48 & 97.55 & 6.20 & 1.67 & 3.3 & -3.48 \\
\hline Step $_{1}$ & 4.88 & 12.74 & 7.48 & 85.72 & 9.54 & 7.21 & 22.4 & -0.31 \\
\hline Step $_{2}$ & 0.43 & 1.91 & 0.29 & 99.58 & 3.95 & 2.19 & 0.9 & -5.99 \\
\hline $\mathrm{Step}_{3}$ & 71.04 & -12.97 & 1.10 & 96.99 & 58.65 & 64.84 & 3.3 & 5.66 \\
\hline Step $_{4}$ & 6.22 & 1.66 & 1.72 & 86.04 & 7.49 & 6.86 & 5.2 & -0.37 \\
\hline Residue & 17.05 & 1.00 & 0.09 & 76.92 & 20.37 & 18.71 & 0.3 & -18.34 \\
\hline
\end{tabular}

